Drug Delivery and Translational Research

\title{
Influence of cholesterol on liposome stability and on in vitro drug release
}

\author{
Maria-Lucia Briuglia ${ }^{1}$, Chiara Rotella ${ }^{2}$, Amber McFarlane $^{1}$, Dimitrios A. Lamprou ${ }^{1 *}$ \\ ${ }^{1}$ Strathclyde Institute of Pharmacy and Biomedical Sciences (SIPBS), University of \\ Strathclyde, 161 Cathedral Street, Glasgow, G4 ORE, Scotland, UK. \\ ${ }^{2}$ Conway Institute of Biomolecular and Biomedical Research, University College Dublin, \\ Belfield, Dublin 4, Ireland.
}

*Corresponding author: dimitrios.lamprou@strath.ac.uk

Keywords: cholesterol, liposomes, controlled release, AFM, mathematical modelling

\begin{abstract}
:
Cholesterol plays a strategic role in liposome composition, however the quantity used to achieve an appropriate formulation has not been yet clarified. Therefore, by screening arrange of lipids and cholesterol ratio, the main aim of this study is to investigate the most suitable amount of cholesterol in lipids in order to prepare stable and controlled drug release vehicles. For the preparation of liposomes, DMPC, DPPC and DSPC phospholipids were used and combined with different molar ratios of cholesterol (e.g. 100\%, 80-20\%, 70-30\%, 60-40\%, $50-50 \%)$. Stability studies were conducted storing the formulations at $37^{\circ} \mathrm{C}$ and $50^{\circ} \mathrm{C}$ for 30 days and by analysing them by AFM, DLS and FT-IR. By detecting the two most stable formulations from the stability results, drug encapsulation and in vitro release studies in PBS were performed by encapsulating Atenolol and Quinine. The release results were validated using a simulation model to ensure the reliability and suitable interpretation of the data. The generated model showed a good correlation between the prediction and the in vitro obtained results. By using 70:30\% ratio (known in literature as 2:1), is possible to reach the most stable formulation to guarantee a controlled and reproducible release for drugs with different physicochemical characteristics and pharmaceutical applications.
\end{abstract}




\section{Introduction}

Liposomes or "lipid bodies" [1] are microstructures composed of a bilayer of natural or synthetic lipids [2], which arrange in polar head group and long hydrophobic tail (e.g. phospholipid or lecithin) forming an amphipathic environment. Moreover, liposomes pack concentrically around the hydrophilic and lipophilic portions being roughly equal obtaining an interior "vacuole" which can serve as a storage compartment for an active agent. The presence of this unique structure is suitable for encapsulation of drugs with different lipophilicities such as strongly lipophilic or hydrophilic drugs, and drugs with intermediate $\log$ P [3], providing solutions for drug delivery. Liposomes have been extensively investigated for drug targeting [4] [5], drug delivery [6] [7], controlled release [8], and increased solubility [9]. However, the major rate-limiting step in the use of this versatile drug delivery system is the physical and chemical stability of the liposome [1]. In phospholipid bilayers two types of chemical degradation reactions can affect the performance of the liposomes, hydrolysis of the ester bonds linking the fatty acids to the glycerol backbone and the peroxidation of unsaturated acyl chains (if present). The oxidation and hydrolysis of lipids may lead to the appearance of short-chain lipids, and then soluble derivatives will form in the membrane which can result a decrease in the quality of liposome products [10]. Moreover, physical processes such as aggregation/flocculation and fusion/coalescence, can affect the shelf life of liposomes due to loss of liposome associated drug and changes in size. It is detectable that the stability can affect the performances of liposomes. Therefore, the control over their physical stability becomes an important prerequisite for the effective use of liposomes as a drug carrier [11], and any change in particle size of carriers can affect targeting, safety and efficacy [1]. It is important to guarantee a good stability of liposomes in terms of rigidity of the lipid bilayer by selecting lipids with a high phase transition temperature $(\mathrm{Tc})$, which is affected by fatty acid side chains, degree of unsaturation, chain length and polar head groups [12]. In this study phosphatidylcholine (PC) liposomes were selected since are lipids with long acyl chain length (DMPC: 14 C; DPPC: 16 C; DSPC: 18 C), unsaturated and consequently with a high transition temperature.

A very important variant in the liposomal structure that could control the stoutness is the content of cholesterol. Numerous studies on the use of cholesterol as a stabilizer were conducted, showing that this steroid can: (1) increase the packing of phospholipid molecules [13], (2) reduce bilayer permeability to non-electrolyte and electrolyte solutes [14], (3) 
improve vesicle resistance to aggregation [15], (4) change the fluidity of intravesicle interactions make them more rigid and sustain in sever shear stress of the lipid bilayer [16], and (5) reduce drug incorporation efficiency [17]. The maximum amount of cholesterol that has been reported and can be incorporated into reconstituted bilayers is widely assumed to be about $50 \mathrm{~mol} \%$, [18] [19] [20]. However, the ratio between cholesterol and lipid used to reach the more stable and more efficient formulation is not well clarified. The most frequently used proportion is a 2:1 ratio (e.g. 2 parts of lipids and 1 part of cholesterol) or 1:1 ratio; however, the reason for this particular ratio is unknown. To understand this preference, this paper will investigate a greater variety of ratios and different storage temperatures by using Atomic Force Microscopy (AFM), Dynamic Light Scattering (DLS), and Fourier Transform Infrared Spectroscopy (FTIR), in order to either confirm that this is the optimum ratio for liposome stability or to find a better alternative. Additionally, after the stability studies, the article will focus the attention on release of two drugs with different physicochemical characteristics that loaded into the two "best" liposome formulations. The drugs selected were Atenolol (A) as hydrophilic model and Quinine (Q) as hydrophobic example. They belong to $\beta$-blocker and antimalarial class respectively providing an interesting study of controlled release for two classes of drugs that largely used and confirming the wide range of liposomes application as drug delivery systems. Furthermore, in order to ensure an accurate interpretation of the results, the drug release studies were validated using a mathematical model, demonstrating its utility in extracting reliable rate constants where different drug bindings can affect the apparent release kinetics.

\section{Materials and Methods}

\subsection{Materials}

The synthetic lipids 1,2-dimyristoyl-sn-glycero-3-phosphocholine (DMPC), 1,2-dipalmitoylsn-glycero-3-phosphocholine (DPPC) and 1,2-Distearoyl-sn-glycero-3-phosphocholine (DSPC) were a gift from Lipoid GmbH (Fig. 1). Cholesterol (CH), Atenolol (A) and Quinine (Q) were obtained from Sigma-Aldrich.

\subsection{Preparation of liposomes}

Liposomes were prepared using the film hydration method as previously described by Bangham et al.[21], by mixing a variety of lipids and using different ratio of lipid/cholesterol (100\% of lipids, $80: 20 \%, 70: 30 \%, 60: 40 \%, 50: 50 \%)$. The mixture of lipid and cholesterol 
was dissolved in chloroform and then it was dried up to a thin lipid film under vacuum using a rotatory evaporator (Heidolph, Germany). For the stability studies the liposomes were prepared without adding drug solution, thus the dry lipid film was rehydrated with phosphate buffered saline (PBS) in order to obtain a lipid suspension. The liposomes used to analyse the drug release were prepared by rehydrating the thin lipid film with the drug solution $(10 \mathrm{mg}$ $\mathrm{mL}^{-1}$ Atenolol and $0.45 \mathrm{mg} \mathrm{mL}^{-1}$ Quinine). The liposome solution in both cases was divided into three Eppendorf tubes and it was sonicated for $30 \mathrm{~min}$ in a VWR ultrasonic cleaner. Following sonication in accordance with literature, the liposome suspensions were left to stand for $1 \mathrm{~h}$ at a temperature higher than the transition temperature of the lipid used in each case (e.g. $40^{\circ} \mathrm{C} \mathrm{DMPC}, 50^{\circ} \mathrm{C} \mathrm{DPPC}, 70^{\circ} \mathrm{C}$ DSPC), in order to anneal any structural defects [22]. The top liquid layer was removed leaving the deposited liposomes at the bottom of the centrifuge tube. In order to conduct the stability studies of formed liposomes the centrifuge product was rehydrated with PBS and was used for liposome characterisation.

\subsection{Liposomes Physicochemical Characterization}

\subsubsection{Dynamic Light Scattering (DLS)}

The size distribution (mean diameter and polydispersity index) and the zeta $(\zeta)$ potential of the liposomes were measured by DLS on a ZetasizerNano-ZS (Malvern Instruments Ltd., UK), which enabled to obtain the mass distribution of particle size as well as the electrophoretic mobility. Measurements were made at $25^{\circ} \mathrm{C}$ with a fixed angle of $137^{\circ}$. Sizes quoted are the z-average mean $(\mathrm{dz})$ for the liposomal hydrodynamic diameter $(\mathrm{nm})$.

\subsubsection{Fourier Transform Infrared Spectroscopy (FTIR)}

All the excipients such as DMPC, DPPC, DSPC, and cholesterol were analysed separately with infrared (IR). The pellets were scanned in an inert atmosphere over a wave number range of 3000-1500 cm $\mathrm{cm}^{-1}$ over 128 scans at a resolution of $4 \mathrm{~cm}^{-1}$ and an interval of $1 \mathrm{~cm}^{-1}$. All FT-IR spectra were recorder on a Nicolet iS10 (ThermoScientific) Smart iTR spectrophotometer and each spectrum was background subtracted.

\subsubsection{Atomic Force Microscopy (AFM)}

A volume of $5 \mu \mathrm{l}$ from each liposome and liposome-cholesterol mixture was placed on a freshly cleaved mica surface (1.5 cm x $1.5 \mathrm{~cm}$; G250-2 Mica sheets 1" x 1" x 0.006"; Agar Scientific Ltd., Essex, UK). Each aliquot was kept on the mica surface for 5 minutes and then 
washed with $100 \mu \mathrm{l}$ of water (Millipore, 18.2 M $\mathrm{cm}$ ). The sample was then air-dried and imaged at once by scanning the mica surface in air under ambient conditions using a Bruker MultiMode8 Scanning Probe Microscope (Digital Instruments, Santa Barbara, CA, USA) operated in tapping mode. AFM measurements were obtained using soft silicon probes (FESP; nominal length $225 \mu \mathrm{m}$, width $28 \mu \mathrm{m}$, tip radius $8 \mathrm{~nm}$, resonant frequency $75 \mathrm{kHz}$, spring constant $2.8 \mathrm{~N} / \mathrm{m}$; Bruker Instruments SAS, Dourdan, France). AFM scans were acquired at a resolution of $512 \times 512$ pixels and produced topographic images of the samples in which the brightness of features increases as a function of height. Typical scanning parameters were as follows: tapping frequency $73 \mathrm{kHz}, \mathrm{RMS}$ amplitude before engage $1 \mathrm{~V}$, integral and proportional gains 0.3 and 0.5 respectively, set point $0.4-0.7 \mathrm{~V}$, and scanning speed $1.0 \mathrm{~Hz}$. Surface roughness (Ra) values were determined by entering surface scanning data into a digital levelling algorithm (Nanoscope Analysis 1.5) and the particle size (diameter) are determined using Nanoscope Analysis 1.5 image processing software which obtained height patterns and cross sections of the lipid nanoparticles. AFM images were collected from two different samples and at random spot surface sampling (minimum of 5 areas) by counting more than 50 liposomes.

\subsubsection{Dialysis dynamic experiment}

Dynamic dialysis is one of the most commonly used methods for the determination of release kinetics from nanoparticles [23]. Prior to the addition of the mixture, the dialysis tube (Millipore, 18.2 $\mathrm{M} \Omega \mathrm{cm}$ ) was placed in boiling water for $30 \mathrm{~min}$ and rinsed with copious amount of water. After the pre-treatment, following a dynamic dialysis procedure described by Joguparthi et al. [24] a volume of $1 \mathrm{~mL}$ of liposomal suspension was added into the dialysis bag tying both ends and suspending the tube in $5 \mathrm{~mL}$ PBS at pH 7.4 [25].

\subsection{Drug release experiment}

Drug release was analysed by extraction of $400 \mu \mathrm{L}$ aliquots of the immersion medium at intervals of $15 \mathrm{~min}, 30 \mathrm{~min}, 1,2,3,4,24,48,72 \mathrm{~h}$ and 7,14 days at $37^{\circ} \mathrm{C}$ and at $7.4 \mathrm{pH}$. To satisfy the perfect-sink conditions and keeping the volume of the solution constant, the supernatant was replaced with fresh PBS pre-equilibrated at $37^{\circ} \mathrm{C}$ at each time point allowing the determination of the diffusion parameters. The amount of drug released at each time point was determined by UV-Vis using a Varian 50 bio UV-visible spectrophotometer at room temperature. The concentration of the drug released from the dialysis tube was determined using a calibration curve of the pure drugs in PBS solutions at the wavelength where showed 
the maximum absorbance (Atenolol: 224nm [26] and Quinine: 250nm [27]).The absorbance was converted into percentage release using a standard curve and experiments were performed in triplicates in order to ensure accuracy.

\subsection{Mathematical Modelling and Simulation}

Data fitting and simulation were done on previously developed mathematical models [24]

[28]. Thus, the considered equation was:

$$
\frac{M_{t}}{M_{\infty}}=k_{1} t^{m}+k_{2} t^{2 m} \mathbf{E q . 1} \text { (Model developed by Peppas and Sahlin) }
$$

Where $t$ represents the time, $k_{1}, k_{2}$ and $m$ are constants. $M_{t} / M_{\infty}$ represents the Fickian diffusional contribution considering the amount of drug released at time $\mathrm{t}$ and infinite time. These parameters were used as the initial input in Igor Pro 6.34A to refine the estimations using an optimization method.

\section{Results and Discussion}

\subsection{Particle size measurements}

The size distribution is a key aspect to consider in the characterization of liposomes. In order to get more detailed and quantitative understanding of the stabilization effect of the different quantities of cholesterol added to the lipids, DLS was used. The temperature selected to conduct the analysis with empty liposomes were $37^{\circ} \mathrm{C}$ as the human body temperature, and $50^{\circ} \mathrm{C}$ that is particularly significant for the antimalarial release study. The stability studies were carried out for 30 days, showing in all experiments the same performances (Figs.2-4), and liposomes prepared without cholesterol (Fig.5) at both temperatures show an unstable but expected behaviour.

Using the film hydration method to manufacture the liposomes no filtration or extrusion procedures [29] were performed for all set of experiments proving values of mean diameter above $200 \mathrm{~nm}$ both with and without cholesterol. Concerning the only lipid formulation, the size range presents the values of mean diameter: DMPC (297.45 $\pm 9.5 \mathrm{~nm})$; DPPC (305.6 \pm $8.6 \mathrm{~nm})$; DSPC (385.2 $\pm 7.8 \mathrm{~nm})$. Regarding the highest mixture of lipid and cholesterol (50$50 \%)$, the size range presents the diameter: DMPC $(292.5 \pm 3.8 \mathrm{~nm})$; DPPC $(289.0 \pm 5.6 \mathrm{~nm})$; DSPC $(362.7 \pm 5.6 \mathrm{~nm})$. 
Looking at the stability, especially for DMPC, the formulation manufactured using only lipid (Fig. 2a), is clearly altered because of the transition temperature (Tc) of 23oC (Table 1). It is obvious to link the unstable performance of DMPC without cholesterol to the temperature chosen to conduct the analyses. At $37^{\circ} \mathrm{C}$ and $50^{\circ} \mathrm{C}$, DMPC liposomes are above the Tc and the bilayer is in a more fluid liquid crystalline phase [34]. Membrane chain melting properties are regulated by the polar surface of the lipid bilayer, reducing the stability and creating a different arrangement in structures where aggregation can occur. In pure DPPC and DSPC the Tc are $41^{\circ} \mathrm{C}$ and $54^{\circ} \mathrm{C}$, respectively, guarantying a more orderly behaviour. Although not ideal (Fig.5 b-c), since the first addition of cholesterol in ratio 80:20 the light scattering showed a clear reduction in size, identifying an average of the diameters for DMPC (268.9 \pm $6.8 \mathrm{~nm})$, DPPC $(255.6 \pm 10.3 \mathrm{~nm})$, and DSPC $(360.6 \pm 6.7 \mathrm{~nm})$. These results demonstrate that by introducing cholesterol into the lipid bilayer, a more stable trend was detected reaching the aim of increasing the state of order. Gradually the amount of cholesterol incorporated in the liposomes was improved causing an increase also in terms of the sizes as in consistent with the literature [16]. Furthermore, looking at the $\zeta$-potential results [DMPC 100\% (0.87 \pm 0.12$)$, DMPC 70\%-30\% (-0.50 \pm 0.13$)$ ], [DPPC 100\% (0.92 \pm 0.16$)$, DPPC $70 \%-30 \%(-0.41 \pm 0.25)]$, and [DSPC 100\% (0.79 \pm 0.11$)$, DSPC 70\%-30\% (-0.71 \pm 0.18$)]$, it is clear that the $\zeta$-potential values are approximately zero for all $100 \%$ lipids samples and converts to negative, improving particles stability when the amount of cholesterol is been increased.

The important rule of cholesterol in liposome formulation is evident from the stability profile of DMPC data. Fig.2 shows an improvement of the chord lengths distribution with the gradually enhancement of cholesterol, which tends to raise the Tc obtaining a range of temperature where the membrane elasticity modulus is much larger [35] [32] and it can affect the liposomes size [37].

For each lipid combinations investigated, the two more stable formulations were chosen to make a further study by using Atenolol and Quinine in order to analyse their drug release. The selected combinations were: DMPC 70:30\% and 50:50\%, DPPC 0:30\% and 60:40\%, and DSPC 70:30\% and 60:40\%. Confirming what the literature asserts, the ratio 70:30\%, which represents the proportion 2:1, is the common mixture in all cholesterol/PC formulations. 


\section{2. $A F M$}

AFM is a rapid, powerful and relatively non-invasive technique that was used in this study to investigate the effects of cholesterol concentration on the liposomes adsorbed on mica surface providing a characterization about shape, morphology and surface properties. Since lipids are soft samples the tapping mode procedure was used; it consists in an intermittent contact between the cantilever and the sample in order to reduce frictional and adhesive forces, which would deform or scrape the sample [38][39].Amplitude images of the PC and PC/cholesterol (ratio 70:30 molar) are shown in Fig 6. However, AFM results for PC/cholesterol molar ratio 50:50 and 60:40 are not reported because the quality of the obtained images did not permitted an appreciable analysis on the individual vesicle. The liposome suspensions incubated with the highest content of cholesterol, in accordance with Liang [40] appeared blurred without show a visible sphericity, providing the suggestion that the best combination to reach a perfect formulation of liposomes is the 70:30ratios. Considering the images carried out from the sample without cholesterol (Fig.6 a,c,e), it is possible to confirm the instability of liposomal formulation on mica surface.

The cloudy area clearly visible in Fig.6c is characterized by a flattened layer of lipids with high viscosity, which can be related to collapse lipids and not well assembled to the rest of the liposomal structure [41]. Moreover, when cholesterol is added to the lipid bilayer (Fig. 6 $b, d, f)$, the images refer spherical and well-defined structure confirming the essential rule of the cholesterol in the liposome formulations in order to increase the state of order. However, knowing from the literature that despite the tapping mode application, liposomes can change their shape once deposited on mica support inducing deformations [42] depending chiefly on the vesicle composition [43], a comparison between AFM results and DLS data was performed in order to validate the analysis (Table 2). In AFM detection, the most common problem is the collapse of lipids, however despite that Kanno et al. [44] demonstrated that the diameter acquired by AFM measurements can be compared with the size obtained using DLS analysis evaluating the differences. According to DLS, AFM reveals a difference in terms of reduction of particle size for lipids modified with cholesterol [45]. Furthermore, the formulation with ratio $70-30 \%$ for each lipid analysed, which shows small standard deviation, suggesting well-assembled structure.

\subsection{FT-IR}

The variation on cholesterol concentrations are causing changes in the liposomal membrane 
structure, producing various frequencies of vibrational modes [46]. Therefore, the characterisation of the liposome formulations using FTIR was performed in order to understand the cholesterol interaction with phospholipids. A comparative study between the pure lipid and formulated liposomes is shown in Fig.7.

The strongest bands at $2920 \mathrm{~cm}^{-1}$ and $2850 \mathrm{~cm}^{-1}$ represent the $\mathrm{CH}_{2}$ symmetric and antisymmetric stretch modes [47], and the peak around $1740 \mathrm{~cm}^{-1}$ characterizes the $\mathrm{C}=\mathrm{O}$ stretch of the ester bond, where the fatty acid chain meets the head group [48]. FTIR spectra have been compared considering the increase of cholesterol amount which changes the detected signal. The gradual addition of cholesterol provides a regular loss of information, showing a disturbed signal in the region clearly detected previously. For each lipid where the added cholesterol was 50\% (DMPC) or 40\% (DPPC and DSPC) the detection of peaks was possible, especially in the region around $1740 \mathrm{~cm}^{-1}$ since cholesterol provides a steric hindrance hiding the $\mathrm{C}=\mathrm{O}$ stretch. These results represent an additional prove that the 70:30 ratio represents the most suitable compromise to increase the stability without alter the lipid composition.

\subsection{Encapsulation efficiency}

After stability and characterisation studies the two drug molecules A and Q were encapsulated in the two most stable detected formulations of DMPC, DPPC and DSPC. The factors that are affecting the encapsulation efficiency (\%EE) of the drug in the liposomes are various and originated from the liposomes and the loaded drug properties [49]. Concerning the encapsulated molecules, the \%EE is affected by hydrophilic or hydrophobic properties, and also the interaction with the membrane bilayer is subjected to changes of different amount of cholesterol. The \%EE was calculated using the formula in Eq.2:

$$
\% E E=\frac{W_{t}}{W_{i}} 100 \mathbf{E q . 2}
$$

Where $\mathrm{W}_{\mathrm{t}}$ is the total amount of drug in the liposomes suspension and $\mathrm{W}_{\mathrm{i}}$ is the total quantity of drug added initially during preparation. Table 3 shows the $\% \mathrm{EE}$ results for all formulation combinations; it can be observed for both drugs (A and Q) that by increasing the incorporated $\%$ of cholesterol the amount of drug loaded it's been reduced. Despite the different physicochemical characteristics of the chosen compounds, the $\% \mathrm{EE}$ results are relatively high for both compounds demonstrating how the film method used to prepare liposomes had been produced a possible link to the polar head for A and to the non-polar chains for Q. 
Previous works had demonstrated a competitive action within the liposomal bilayers between drugs and amount of cholesterol on the incorporation of drugs [50] [51]. The literature reports the capacity of cholesterol to increase the hydrophobicity of the membranes, which can favour the inclusion of hydrophobic molecules [52]. However, it must be considered the conflicting fact that cholesterol favours to accommodate into the lipophilic bilayer structure, which is represented by lipid chains [17]. Cases with high presence of cholesterol cause limited space for $\mathrm{Q}$ accommodation [Fig.8, a] due to the steric hindrance provided by the steroid reducing the \%EE.

Cholesterol is been affected also by the hydrophilic drug encapsulation, despite the interaction that occurs with the phosphate heads, which can be explained as a steric hindrance reason. Increasing the amount of cholesterol such as DMPC or DPPC 50-50\% and DSPC 60$40 \%$, the chosen hydrophilic drug (A) will have limited "binding space" where it can be linked to the drug delivery. Moreover, when the formulation is prepared using a reduced quantity of cholesterol, $30 \%$ rather than $50 \%$ or $40 \%$, the $\%$ EE value was increased. The results present encapsulation efficacy of $90 \%$ for $\mathrm{A}$ and $88 \%$ for $\mathrm{Q}$, demonstrating how a reproducible high level of loaded drugs can be reached using a molar ratio of 70-30\%, which represents a good equilibrium between flexibility furnished by lipids and the stability that ensured by cholesterol.

\subsection{Drug release}

The drug release is illustrated in Fig. 9 as a plot of mass released fraction $\left(\mathbf{M}_{t} / \mathbf{M}_{\infty}\right)$ vs time (t).The drug release was measured for A and Q using the following formulation:DMPC 70:30 and 50:50 (Fig.9a), DPPC 70:30 and 50:50 (Fig.9b), and DSPC 70:30 and 50:50 (Fig.9c).

Despite the different physicochemical characteristics of the two studied drugs (one hydrophilic and one hydrophobic) and the different lipid/ cholesterol combinations, common performances were detected for each type of lipids.1) The hydrophilic drug (A) is released faster than hydrophobic drug $(\mathrm{Q}), 2)$ the formulation with the higher amount of cholesterol when A was loaded had recorded the faster release, 3) the combination with a higher amount of cholesterol when Q was added had shown the slower release, and 4) an initial burst release was observed as expected at an incubation temperature of $37^{\circ} \mathrm{C}[53]$. 
The analysis of the drug delivery was furthermore conducted comparing drug release, particle size and content of cholesterol, underling how cholesterol affects both stability and drug release performances (Fig.10).

For all liposomes, the reduction of cholesterol showed a decrease of particle size and different trend on drug release. These performances can be traced to the physicochemical properties of the selected organic compounds and to the competitive action between drugs and cholesterol [17]. As explained in section 3.4, cholesterol affects the release of A, since the hydrophilic drug interact with the head of liposome. Increasing the cholesterol quantity (DMPC 50-50\%, DPPC 60-40\% and DSPC 60-40\%), A will have less encapsulated amount and the release will be faster. On the other hand, considering the location of the hydrophobic drug $(\mathrm{Q})$ which interacts with the long chain of each type of lipids, the augmented presence of cholesterol will cause a less encapsulation efficacy and a reduced release action since the steric hindrance. In accordance with drug release results, the best combination of lipids and cholesterol is represented by $70-30 \%$ reaching a sustained, controlled and reproducible release. The release rates were measured in sink conditions; otherwise the evaluation of the drug release from liposomal dispersions will be problematic due to the dependence of the analysis from the test conditions [54]. In the traditional dialysis technique, the carrier is suspended in a small volume of continuous phase and separated from a sink solution by permeable dialysis membrane [55] providing a physical separation between nanoparticles and free drug at various time points during the kinetic study. The validation of the drug release model was obtained making assumptions to justify the mathematical model used, for example: 1) analysis is based on one-dimensional diffusion, thus edge effects must be negligible; 2) the suspended drug is in a fine state that particles are much smaller in diameter than the thickness of the system; 3) the diffusivity of the drug is constant; 4) perfect sink conditions are maintained; and 5) the appearance of drug in the "sink" is the result of the diffusion from the nanoparticles followed by diffusion across the dialysis membrane (though it is generally treated as a simple first order process). Considering all these assumption, using Eq.1 and Igor Pro 6.34A, a model validation was performed by comparing the predicted drug release with experiment data (Fig.11).

Results demonstrated a good correlation between the measured drug release profile and the prediction, indicating the validity of the model. The mathematical modelling provides a scientific tool to verify the design parameters on the resulting drug release kinetics. 


\section{Conclusions}

The main objective of this study was to investigate the best combination of lipids / cholesterol ration for the formulation of liposomes in order to achieve an appropriate stability profile, and sustained and controlled drug release. Using three types of lipids (DMPC, DPPC and DSPC) and different amounts of cholesterol (e.g. 50\%, 40\%, 30\%, 20\%, and 0\%), a wide range of formulations were prepared. Storing the formed liposomes under two different temperatures (e.g. $37^{\circ} \mathrm{C}$ and $50^{\circ} \mathrm{C}$ ) the stability of the formulations was tested and it was demonstrated by DLS and AFM that cholesterol is required in order to provide a wellorganized structure and a stable formulation. Using the stability results as criteria of choose, the two most stable formulations for each lipid used [DMPC (50-50\% and 70\%-30\%), DPPC (60-40\% and 70\%-30\%) and DSPC (60-40\% and 70-30\%)].Then Atenolol and Quinine were added proving an additional tool to understand the influence of cholesterol in drug release studies. A validation of the drug release using a mathematical model was obtained ensuring the proper interpretation of the data. The generated model showed a good correlation between the prediction and the in vitro results for each formulation investigated. DLS, AFM, \%EE and drug release analysis concluded that the $2: 1$ ratio (70\% of lipids and $30 \%$ of cholesterol) is the most suitable combination in terms of characterisation and as the most flexible formulation, in order to permit the release of drugs with different physiochemical characteristics. This work has confirmed the preference of literature using 2:1 as molar ratio in liposomes formulation and has also added extra information and explanations ensuring a rational understanding on the reasons that the particular combination represents the most frequently used.

Acknowledgments The authors would like to acknowledge the EPSRC Centre for Innovative Manufacturing in Continuous Manufacturing and Crystallisation (CMAC) for using their facilities.

Conflict of interest The authors stated that there are no conflicts of interest. 
Drug Delivery and Translational Research

\section{References:}

[1] Yadav AV, Murthy MS., Shete AS., Sfurti, S. Stability Aspects of Liposomes. Ind. J. Pharm. Edu. Res. 2011; 45: 402-413.

[2] Sharma A, Sharma US. Review: liposomes in drug delivery: progress and limitations.Int. J. of Pharmaceuties. 1997; 154: 123-140.

[3] Immordino ML, Dosio F, CattelL.Stealth liposomes: review of the basic science, rationale, and clinical applications, existing and potential. Int. J. Nanomedicine. 2006; 1: 297315 .

[4] Fattal E, Couvreur P, Dubernet C. "Smart" delivery of antisense oligonucleotides by anionic Ph-sensitive liposomes time. Adv. Drug Deliv. Rev. 2004; 56: 931-946.

[5] Maruyama K, Ishida O, Takizawa T. Possibility of active targeting to tissue with liposomes. Adv. Drug Deliv. Rev. 1999; 40: 89102.

[6] Bouwstra JA, Honneywell-Nguyen PL. Skin structure and mode of action of vesicles. Adv. Drug Deliv. Rev. 2002; 54: S41-S55.

[7] Kaur PI, Garg A, Singla KA. Vesicular systems in ocular drug delivery: an overview. Int. J. Pharm. 2004; 269: 1- 14.

[8] Barber R, Shek P. Liposomes as a topical ocular drug delivery system. A Rolland (Ed) Pharmaceutical particulate carries. Marcel Dekker, New York NY. 1993. pp. 1-20.

[9] Barry BW. Novel mechanism and devices to enable successful transdermal drug delivery. Eur. J. Pharm. sci. 2001; 14: 101-114.

[10] Xue YY. Modern pharmaceutics (Ping Q. N.). China Med-Tech Science Beijing. 1995; 588-62.

[11] Tseng LP, Liang HJ, Chung TW, Huang YY, Liu DZ. Liposomes incorporated with cholesterol for drug release triggered by magnetic field. Journal of medical and biological Engineering. 2007; 27: 29-34

[12] Ceh B, Lasic DD. A rigorous theory of vesicle loading. Longmuir II. 1995; 1141:33563364.

[13] Demel RA, De Kruyff B. The function of sterols in membranes. BiochimBiophysActa. 1976; 457: 109-132.

[14] Papahadjopoulos D, Jacobson K, Nir S, Isac T. Phase transitions in phospholipid vesicles. Fluorescence polarization and permeability measurements concerning the effect of temperature and cholesterol. BiochimBiophys. 1973; 311:330-48.

[15] Virden JW, Berg JC. NaCl-induced aggregation of dipalmitoylphosphatylglycerol small unilamellar vesicles with varying amounts of incorporated cholesterol. Langmuir. 1992; 8: 1532-1537.

[16] Liu DZ, Chen WY, Tasi LM, Yang SP.Microcalorimetric and shear studies on the effects of cholesterol on the physical stability of lipid vesicles. Colloids Surfaces. 2000, 172: 57-67. 
[17] Mohammed AR, Weston N, Coombesa AGA, Fitzgerald M, Perrie Y. Liposome formulation of poorly water soluble drugs: optimisation of drug loading and ESEM analysis of stability. Int J Pharmaceutics. 2004; 285: 23-34

[18] Needham D, Nunn RS. Elastic deformation and failure of lipid bilayer membranes containing cholesterol. Biophys J. 1990; 58:997-1009.

[19] Gregoriadis G, Davis C. Stability of liposomes in vivo and in vitro is promoted by their cholesterol content in the presence of blood cells. BiochimBiophys Res Commun. 1979; 90: 1287-1293.

[20] Kirby C, Gregoriadis G. Effect of the cholesterol content of small unilamellar liposomes on their stability in vivo and in vitro. Biochem J. 1980; 186: 591-598.

[21] Bangham A D, Standish MM, Watkins JC. Diffusion of univalent ions across the lamellae of swollen phospholipids. J. Mol. Biol. 1965; 13: 238-252.

[22] Fatouros D, Gortzi O, Klepetsanis P, Antimisiaris SG, Stuart MCA, Brisson A, Ioannou PV. Preparation and properties of arsonolipid containing liposomes. Chemistry and Physics of Lipids. 2001; 109: 75-89.

[23] Modi S, Bradley AD. Determination of Drug Release Kinetics from Nanoparticles: Overcoming Pitfalls of the Dynamic Dialysis Method, Mol. Pharmaceutics. 2013; 10: 3076-3089.

[24] Joguparthi V, Xiang TX, Anderson BD. Liposome transport of hydrophobic drugs: Gel phase lipid bilayer permeability and partitioning of the lactone form of a hydrophobic camptothecin, DB-67. Journal of Pharmaceutical Sciences. 2008; 97: 400-420.

[25] Katrin K, Christel C, Muller G. Diclofenac release from phospholipid drug system and permeation through excised human stratum corneum. Int. J. Pharm.1995; 125: 231-242.

[26] Bari S, Sathe S, Jain P, Surana S. Spectrophotometric method for simultaneous estimation of atenolol in combination with losartan potassium and hydrocholorothiazide in bulk and tablet formulation. J. Pharm.Bioallied Sci. 2010; 2: 372-375.

[27] Cheon T, Cheong BS, Cho HG, Kim JH, Kim KS. Quinine Assay with Home-Built UVLED Fluorometer: Quantitative Analysis, Photo-Bleaching, Fluorescence Quenching, and Urine Analysis. Journal of the Korean Chemical Society. 2012; 5: 577-582.

[28] Peppas A, Sahlin JJ. A simple equation for the description of solute release. III. Coupling of diffusion and relaxation, Int. J. Pharm. 1989; 57:169-172.

[29] Berger N,Sachse A, Bender J, Schubert R, Brandl M. Filter extrusion of liposomes using different devices: comparison of liposome size, encapsulation efficiency, andprocess characteristics. International Journal of Pharmaceutics.2001; 223: 55-68.

[30] Taly A, Baciou L, Sebban P. The DMPC lipid phase transition influences differently the first and the second electron transfer reactions in bacterial reaction centers. Febs letters. 2002; 532: 91-96. 
[31] Schubert T, Schneck E, Tanaka M. First order melting transitions of highly ordered dipalmitoyl phosphatidylcholine gel phase membranes in molecular dynamics simulations with atomistic detail. J. Chem. Phys. 2011; 135: 055105.

[32] Kučerka N, Nieh MP, KatsarasJ. Fluid phase lipid areas and bilayer thicknesses of commonly used phosphatidylcholines as a function of temperature. Biochimica et BiophysicaActa. 2011; 1808: 2761-2771

[33] Hansch C, Leo A, Hoekman DH. Exploring QSAR, Fundamentals and Application in Chemistry and Biology. American Chemical Society: Washington DC, USA, 1995.

[34] Cevc G. How membrane chain melting properties are regulated by the polar surface of the lipid bilayer. Biochemistry. 1987; 26: 6305-6310.

[35] Hodzic A, Rappolt M, Amenitsch H, Laggner P, Pabst G. Differential modulation of membrane structure and fluctuations by plant sterols and cholesterol. Biophys. J. 2008; 94: 3935-3944.

[36] Henriksen J, Rowat AC, Brief E, Hsueh YW, Thewalt JL, Zuckermann MJ, Ipsen JH. Universal behavior of membranes with sterols. Biophys. J. 2006; 90: 1639-1649.

[37] Yi Z, Nagao M, Bossev DP. Bending elasticity of saturated and monosaturated phospholipid membranes studied by neutron spin echo technique. J. Phys. Condens. Matter. 2009; 21: 155104 .

[38] Albrecht TR, Grutter P, Horne D, Rugard D. Frequency modulation detection using high-Q cantilevers for enhanced force microscope sensitivity. J Appl Phys. 1991; 69: 668673.

[39] Zhong Q, Inniss D, Kjoller K, Elings VB. Fractured polymer/silica fiber surface studied by tapping mode atomic force microscopy. Surface Sci Lett. 1993; 290: 688-692.

[40] Liang X, Mao G, Ng SKY. Mechanical properties and stability measurement of cholesterol-containing liposome on mica by atomic force microscopy. Journal of Colloid and Interface Science. 2004; 278: 53-62.

[41] Ruozi B, Belletti D, Tombesi A, Tosi G, Bondioli L, Forni F, Vandelli MA. AFM, ESEM, TEM, and CLSM in liposomal characterization: a comparative study. International Journal of Nanomedicine. 2011; 6: 557-563.

[42] Jass J, Tjarnhage T, Puu G. From liposomes to supported, planar bilayer structures on hydrophilic and hydrophobic surfaces: An atomic force microscopy study. Biophys J. 2000; 79: $3153-3163$.

[43] Ruozi B, Tosi G, Forni F, Fresta M, Vandelli MA. Atomic force microscopy and photon correlation spectroscopy: two techniques for rapid characterization of liposomes. Eur J Pharm Sci. 2005; 25: 81-89. 
[44] KannoT,Yamada T, IwabukiH,TanakaH,Kuroda S, Tanizawa K, Kawai T. Size distribution measurement of vesicles by atomic force microscopy. Anal. Biochem. 2002; 309, 196.

[45] Onyesom I, Lamprou DA, Sygellou L, Owusu-Ware S, Antonijevic M, Chowdhry BZ, Douroumis D. Sirolimus Encapsulated Liposomes for Cancer Therapy: Physicochemical and Mechanical Characterization of Sirolimus Distribution within Liposome Bilayers. Mol. Pharmaceutics. 2013; 10, 4281-4293.

[46] Casal HL, Mantsch HH. Polymorphic phase behavior of phospholipid membranes studied by infrared spectroscopy. Biochim. Biophys. Acta. 1984; 779: 381-401.

[47] Severcan F, Sahin I, Kazanci N. Melatonin strongly interacts with zwitterionic model membranes - evidence from Fourier transform infrared spectroscopy and differential scanning calorimetry. BBA Biomembranes. 2005; 1668: 215-222.

[48] Jiang CH, Gamarnik A, Tripp CP. Identification of lipid aggregate structures on $\mathrm{TiO}_{2}$ surface using headgroup IR bands. J. Phys. Chem. B. 2005; 109: 4539-4544.

[49] Nii T, Ishii F. Encapsulation efficiency of water-soluble and insoluble drugs in liposomes prepared by the microencapsulation vesicle method. International Journal of Pharmaceutics. 2005; 298: 198-205.

[50] Balasubramanian SV, Straubinger RM.Taxol-lipid interactions: taxol-dependent effects on the physical properties of model membranes. Biochemistry. 1994; 33: 8941.

[51] Needham D, Sarpal RS. Binding of paclitaxel to lipid interfaces: correlations with interface compliance. J. Liposome Res. 1998; 8: 147-163.

[52] Subczynski WK, Wisniewska A, Yin JJ, Hyde JS, Kusumi A. Hydrophobic barriers of lipid bilayer membranes formed by reduction of water penetration by alkyl chain unsaturation and cholesterol. Biochemistry. 1994; 33: 7670-7681.

[53] Hua S. Comparison of in vitro dialysis release methods of loperamide-encapsulated liposomal gel for topical drug delivery. International Journal of Nanomedicine. 2014; 9: 735744.

[54] Saarinen-Savolainen P, Jarvinen T, Taipale H, Urtti A. Method for evaluating drug release from liposomes in sink conditions. International Journal of Pharmaceutics. 1997; 159: 27-33.

[55] Yerushalmi N, Margalit R. Bioadhesive, collagen modified liposomes: molecular and cellular level studies on the kinetics of drug release and on binding to cell monolayers. Biochim. Biophys. Acta. 1994; 1189: 13-20. 
Drug Delivery and Translational Research

\section{Table Captions:}

Table 1. Summary of the key features of the materials that have been used.

Table 2. Particle size results from DLS and AFM.

Table 3. Encapsulation Efficacy of Atenolol and Quinine in each lipid formulation.

\section{Figures Captures:}

Fig. 1. Chemical structures of the compounds used: a) DMPC, b) DPPC, c) DSPC, d) Cholesterol, e) Atenolol (A), and f) Quinine (Q).

Fig. 2. Size distribution of DMPC liposomes with different lipid/cholesterol ratiounder different temperature conditions: a) $100 \%$ lipid, b) $80 \%-20 \%$, c) $70 \%-30 \%$, d) $60 \%-40 \%$, and e) $50 \%-50 \%$. In all graphs the continuous line displays the size distribution at $37^{\circ} \mathrm{C}$ and the dashed line shows the size distribution at $50^{\circ} \mathrm{C}$.

Fig. 3. Size distribution of DPPC liposomes with different lipid/cholesterol ratio under different temperature conditions: a) 100\% lipid, b) 80\%-20\%, c) 70\%-30\%, d) 60\%-40\%, and e) $50 \%-50 \%$. In all graphs the continuous line displays the size distribution at $37^{\circ} \mathrm{C}$ and the dashed line shows the size distribution at $50^{\circ} \mathrm{C}$.

Fig. 4. Size distribution of DSPC liposomes with different lipid/cholesterol ratio under different temperature conditions: a) $100 \%$ lipid, b) $80 \%-20 \%$, c) $70 \%-30 \%$, d) $60 \%-40 \%$, and e) $50 \%-50 \%$. In all graphs the continuous line displays the size distribution at $37^{\circ} \mathrm{C}$ and the dashed line shows the size distribution at $50^{\circ} \mathrm{C}$.

Fig. 5. Size distribution of liposomes prepared without cholesterol: a) DMPC, b) DPPC and c) DSPC.

Fig. 6. AFM images of a) DMPC, b) DMPC-Cholesterol (70-30), c) DPPC, d) DPPCCholesterol (70-30), e) DSPC, and f) DSPC-Cholesterol (70-30).

Fig. 7. FTIR spectra of a) DMPC, b) DPPC, and c) DSPC.

Fig. 8. Schematic model for the encapsulation efficacy of Atenolol (a) and Quinine (b).

Fig. 9. Drug release through dialysis tube of DMPC (a), DPPC (b), and DSPC (c).

Fig. 10. Average particle size (bars, left axis) and \% drug release after 2 weeks (dots, right axis) of different liposome composition.

Fig. 11. Validation of drug release from DMPC (a), DPPC (b), and DSPC (c), using mathematical modelling. 
Drug Delivery and Translational Research

Table 1

\begin{tabular}{cccc}
\hline Molecules & Purity & Temperature lipid transition & $\log \mathbf{P}$ \\
\hline DMPC & $\geq 98 \%$ & $23^{\circ} \mathrm{C}[30]$ & - \\
DPPC & $\geq 98 \%$ & $41^{\circ} \mathrm{C}[31]$ & - \\
DSPC & $\geq 98 \%$ & $54^{\circ} \mathrm{C}[32]$ & - \\
Cholesterol & $\geq 99 \%$ & - & - \\
Atenolol & $\geq 98 \%$ & - & $0.16[33]$ \\
Quinine & $\geq 98 \%$ & - & $3.44[33]$ \\
\hline
\end{tabular}


Drug Delivery and Translational Research

Table 2

\begin{tabular}{cccc}
\hline Lipid & Formulation (lipid / cholesterol) & DLS / nm & AFM / nm \\
\hline \multirow{2}{*}{ DMPC } & $100 \%$ & $297 \pm 9.7$ & $267 \pm 4.9$ \\
& $70-30 \%$ & $273 \pm 2.9$ & $251 \pm 4.1$ \\
DPPC & $100 \%$ & $305 \pm 8.6$ & $284 \pm 6.6$ \\
& $70-30 \%$ & $270 \pm 1.9$ & $230 \pm 2.2$ \\
DSPC & $100 \%$ & $388 \pm 5.3$ & $301 \pm 3.6$ \\
& $70-30 \%$ & $367 \pm 2.9$ & $247 \pm 1.8$ \\
\hline
\end{tabular}


Drug Delivery and Translational Research

Table 3

\begin{tabular}{cccc}
\hline Lipid & Formulation (lipid / cholesterol) & $\begin{array}{c}\text { Atenolol } \\
\text { \% EE }\end{array}$ & $\begin{array}{c}\text { Quinine } \\
\text { \% EE }\end{array}$ \\
\hline \multirow{2}{*}{ DMPC } & $70-30 \%$ & 90.15 & 88.13 \\
& $50-50 \%$ & 87.73 & 84.72 \\
\multirow{2}{*}{ DPPC } & $70-30 \%$ & 90.15 & 88.53 \\
& $60-40 \%$ & 88.60 & 84.07 \\
\multirow{2}{*}{ DSPC } & $70-30 \%$ & 91.56 & 87.54 \\
& $60-40 \%$ & 88.78 & 85.60 \\
\hline
\end{tabular}


Fig. 1
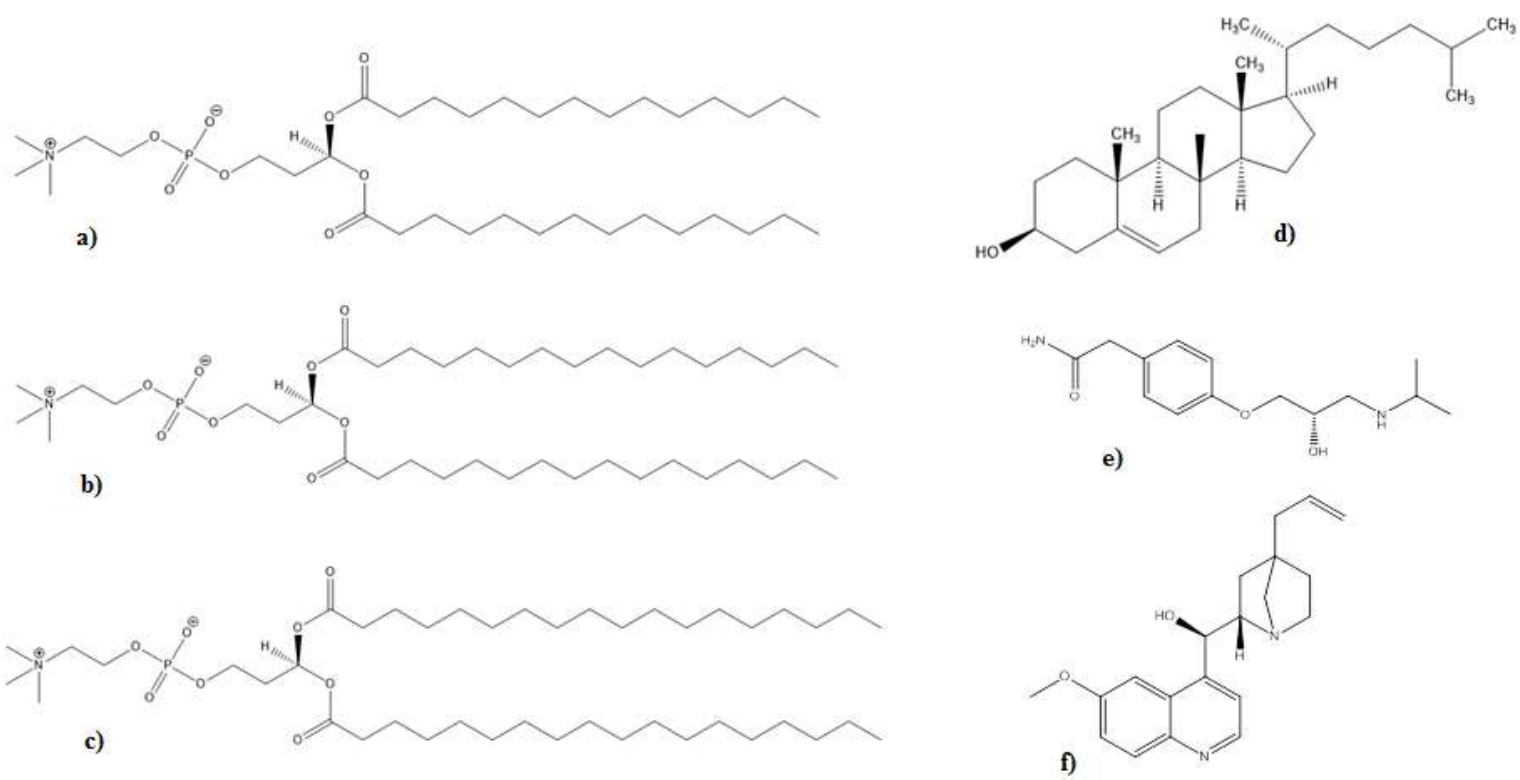
Drug Delivery and Translational Research

Fig. 2
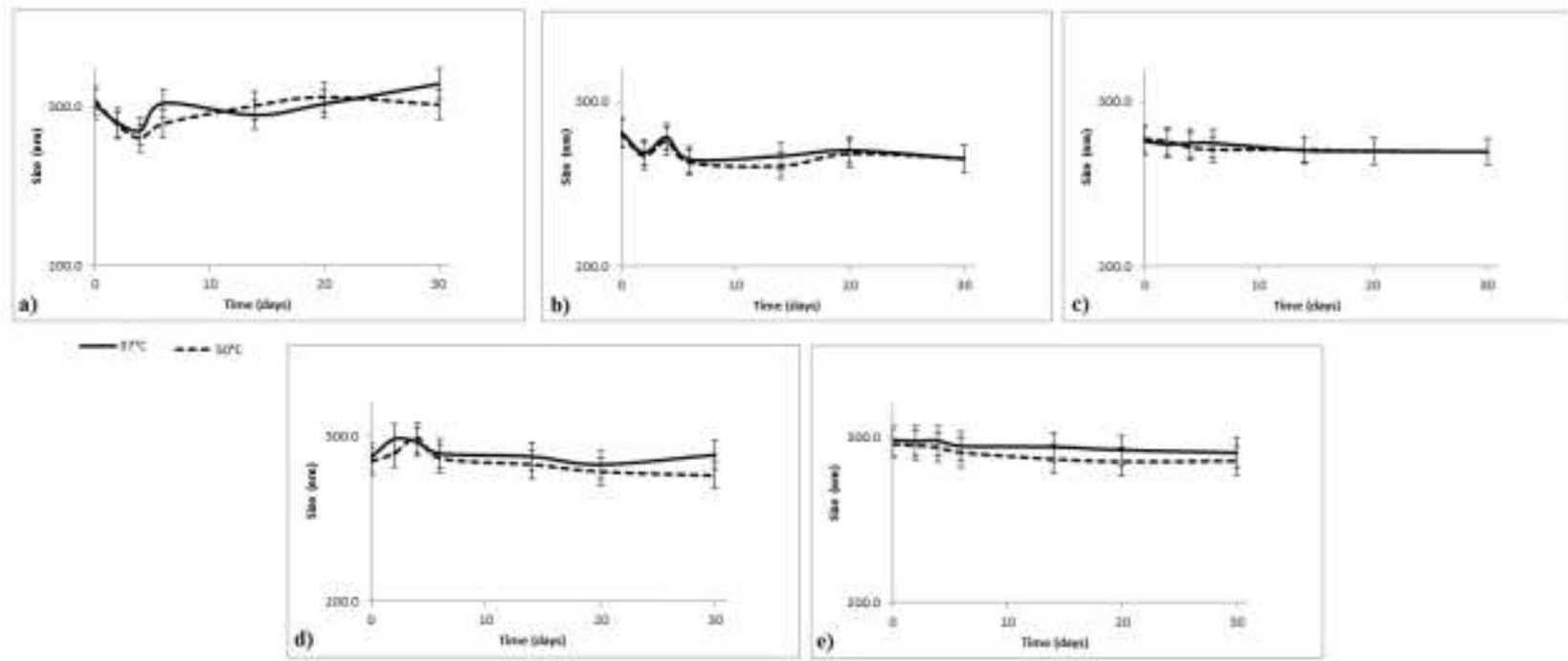
Drug Delivery and Translational Research

Fig. 3

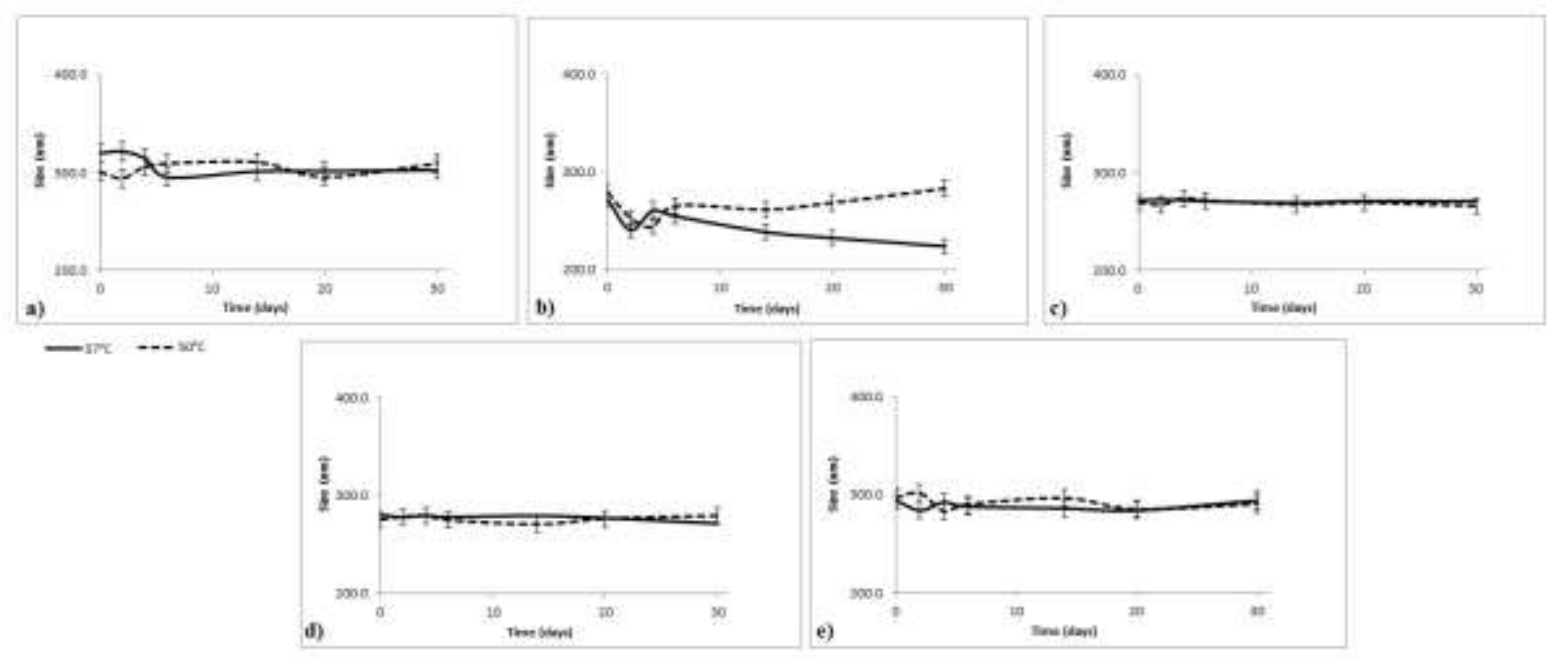


Drug Delivery and Translational Research

Fig. 4

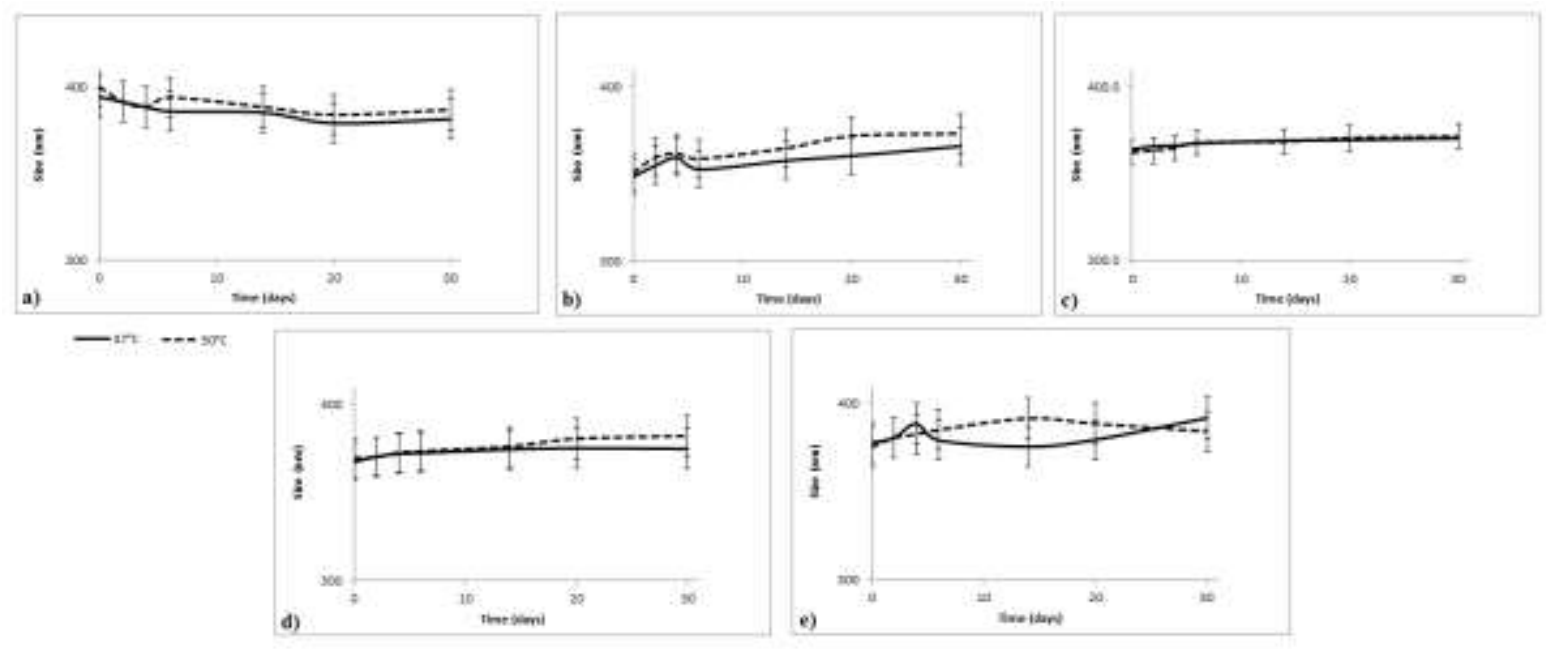


Drug Delivery and Translational Research

Fig. 5
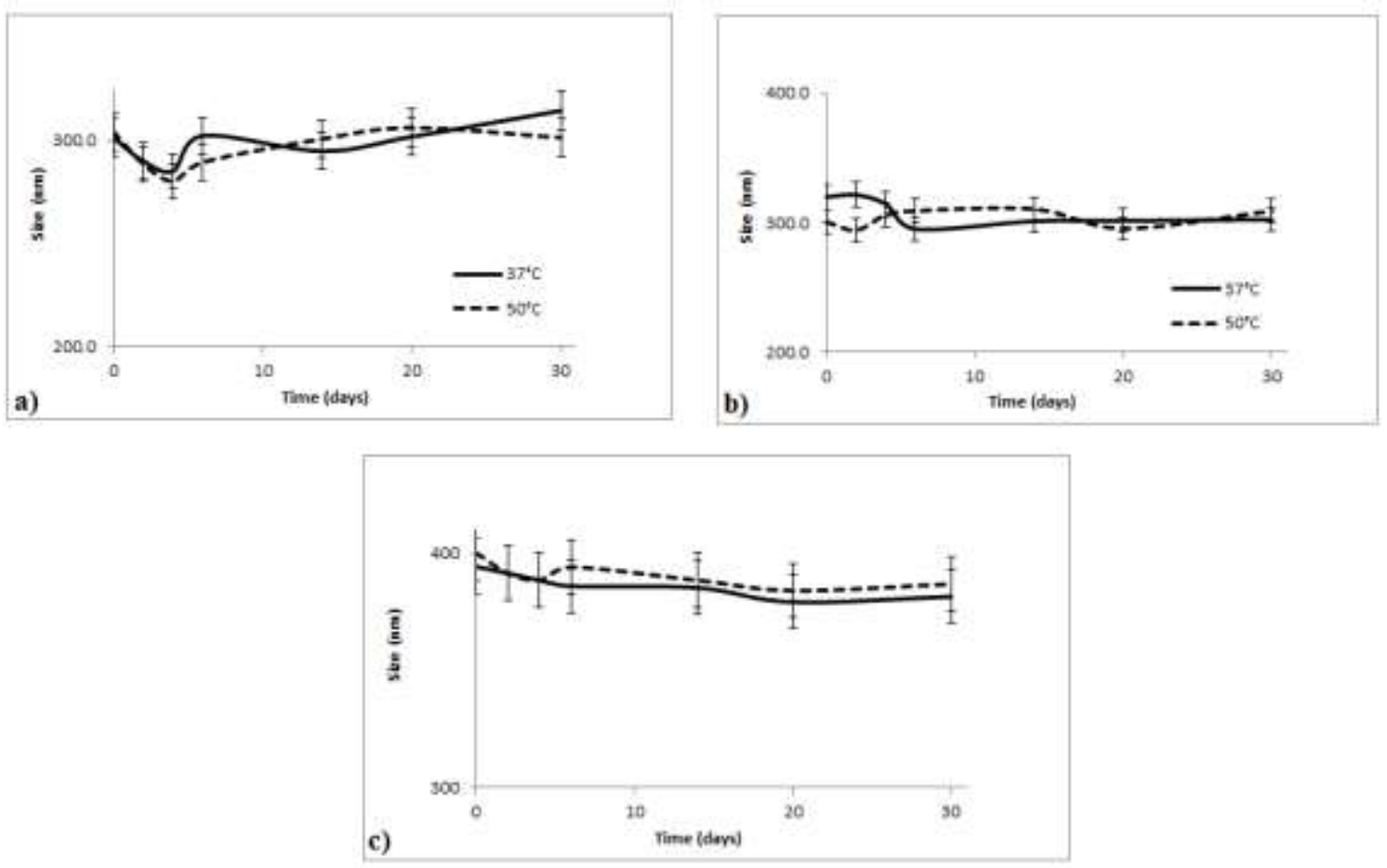
Drug Delivery and Translational Research

Fig. 6

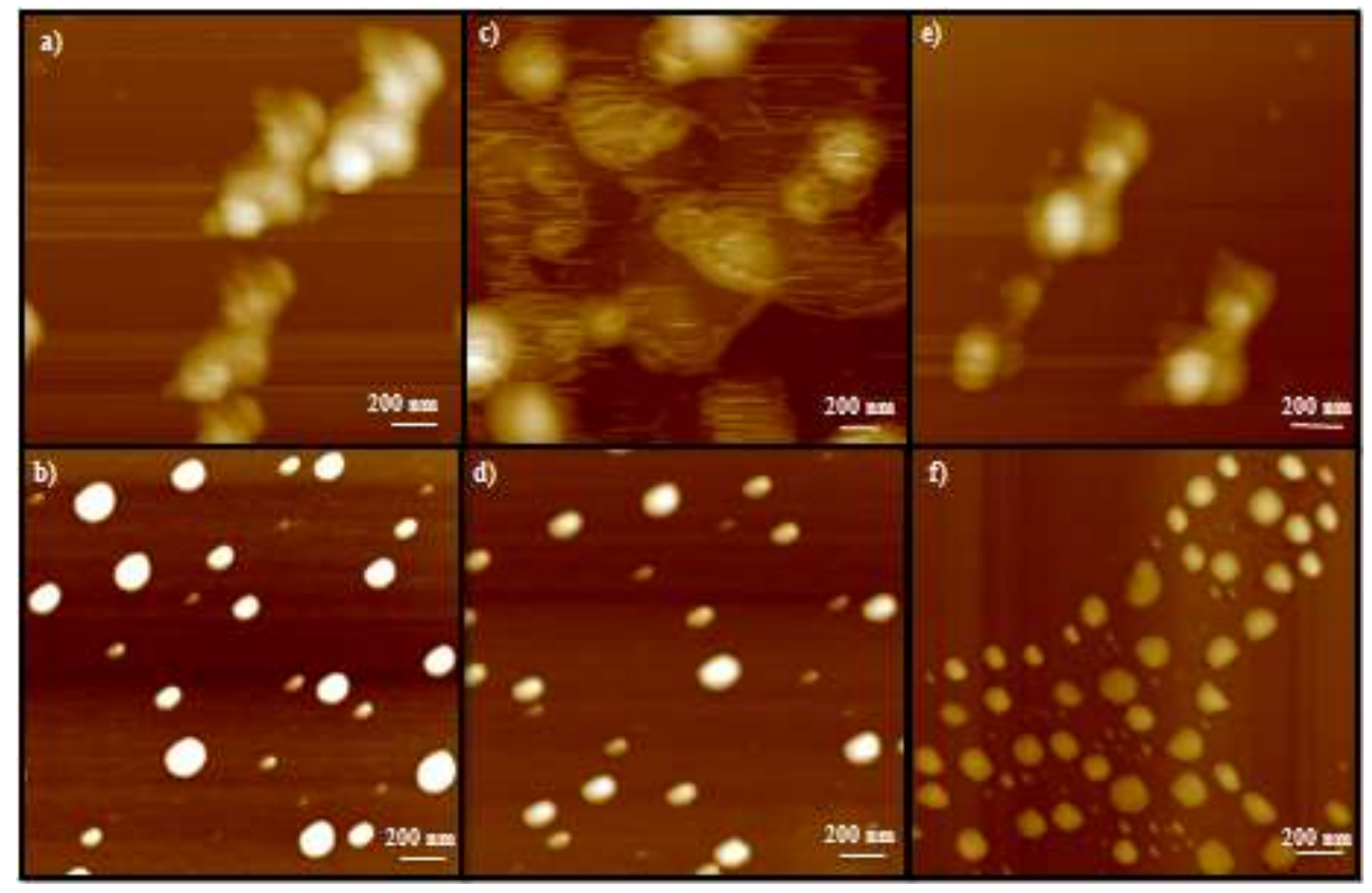


Drug Delivery and Translational Research

Fig.7
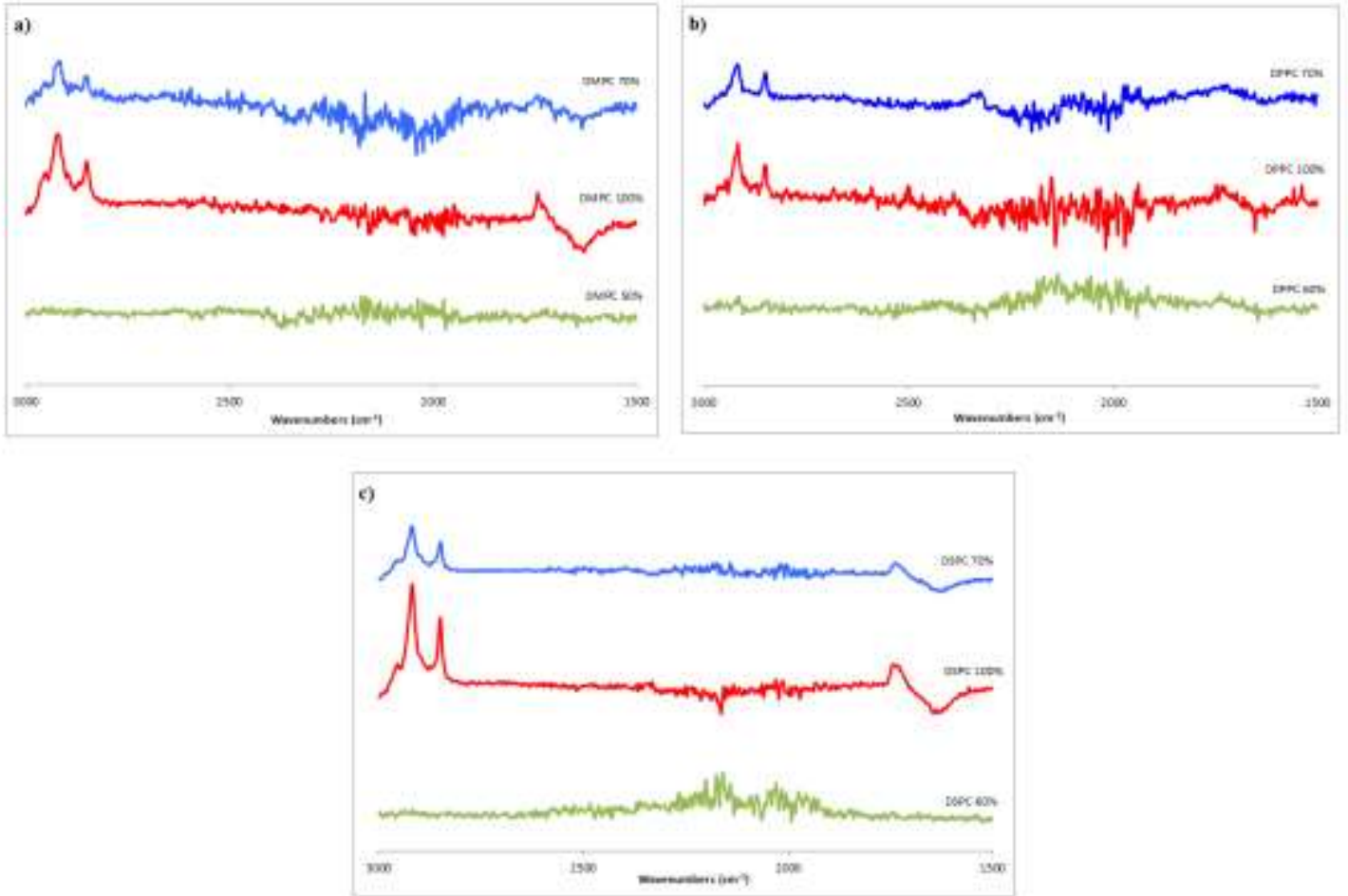
Drug Delivery and Translational Research

Fig. 8

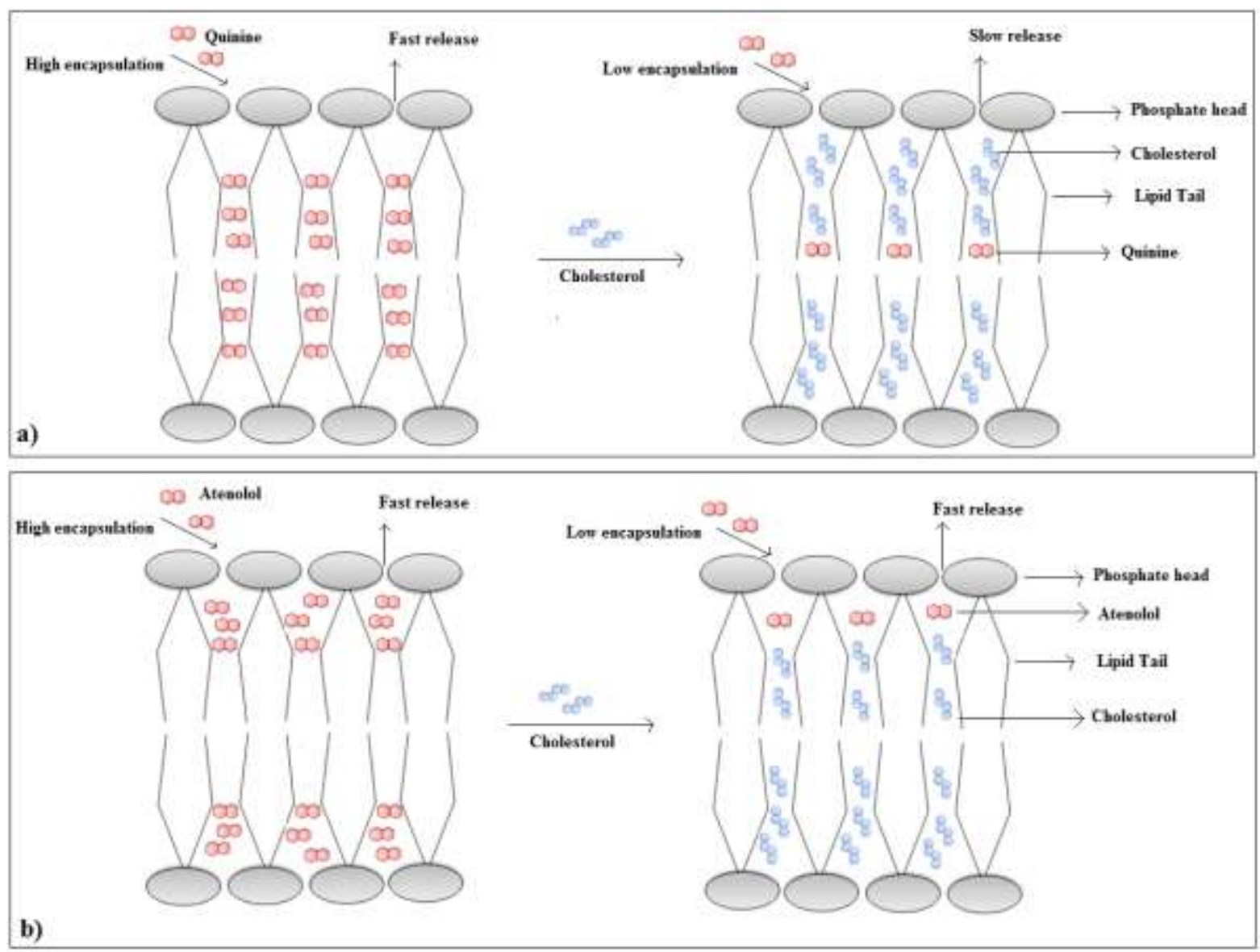


Drug Delivery and Translational Research

Fig. 9
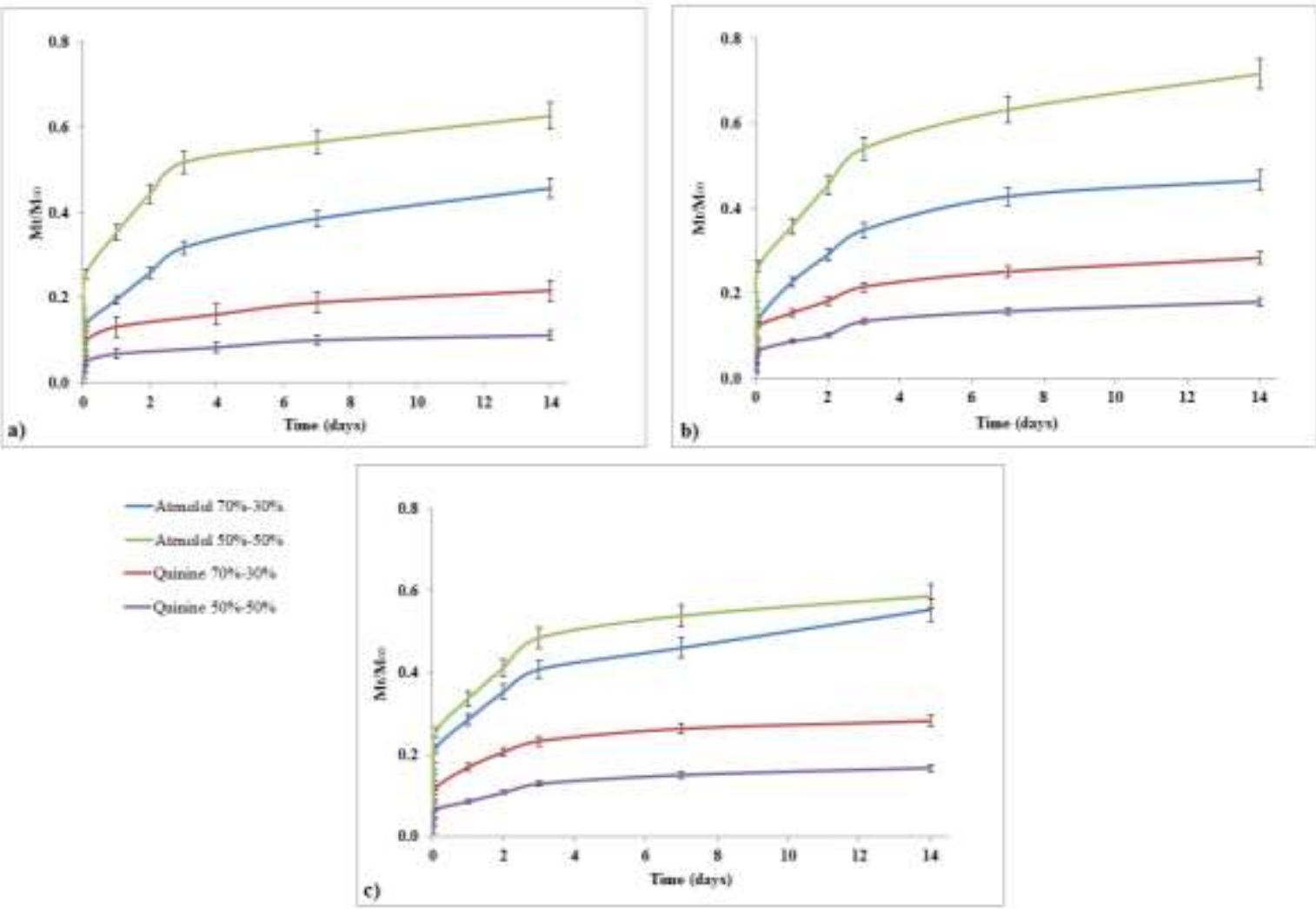
Drug Delivery and Translational Research

Fig. 10

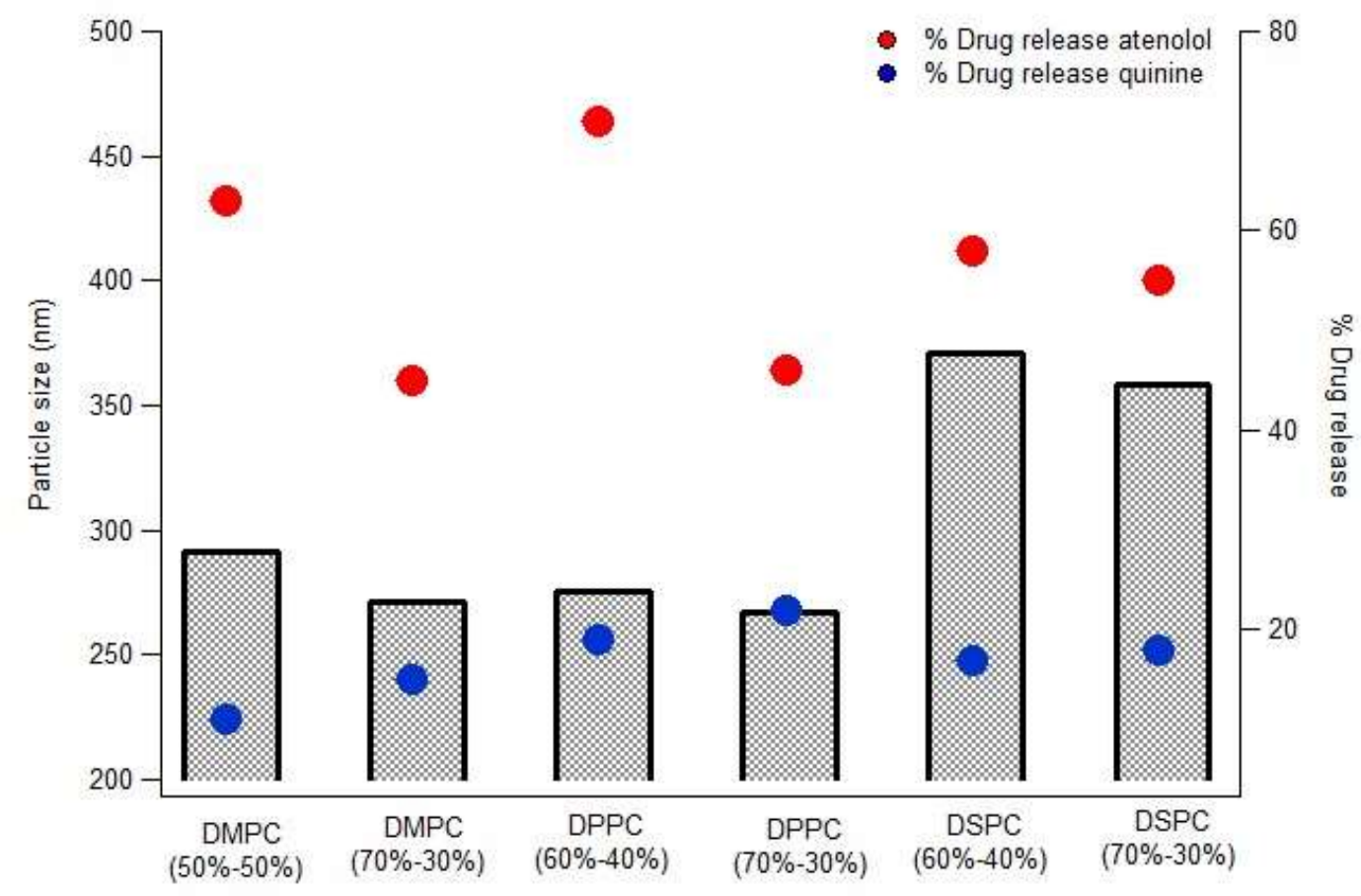


Drug Delivery and Translational Research

Fig. 11
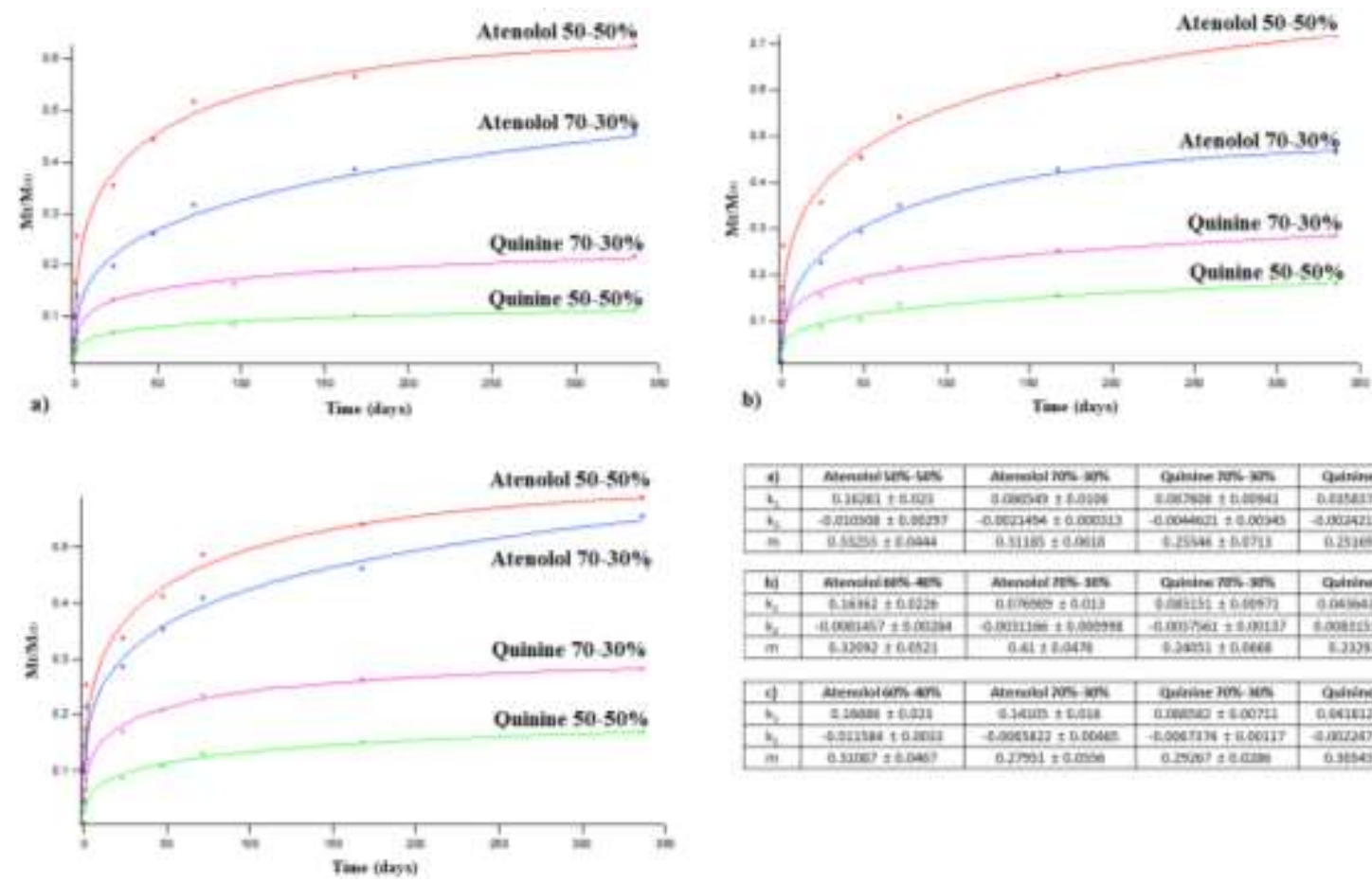

\begin{tabular}{|c|c|c|c|c|}
\hline A) & Atendite bes tes & Aimsolinh an & 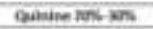 & qdinimen uen bis \\
\hline 2 & Eapen tans & scowon tratos & 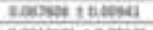 & Dasuer temoss \\
\hline 5 & Solosoo +6.0628 & $-\operatorname{abchust}=0.000013$ & exolveri 20.0000 & 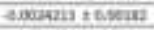 \\
\hline$=$ & 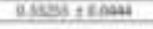 & a.mon $=$ conin & 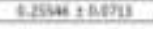 & e2suetowe \\
\hline ti & Giendinitus ats & Alendid IIS IS & 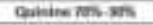 & atrinetes as \\
\hline$s_{1}$ & Anew thelas & 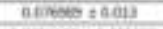 & Dainh towen & onstali tsapen \\
\hline 5. & 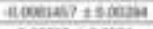 & conine i coost & 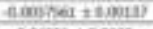 & 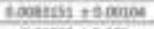 \\
\hline$m$ & LNow r reotal & Q44) & czami treeu & $5208+030$ \\
\hline 9 & sterwablan on & Anewablins on & Quabiare in wa & Qditunetes as \\
\hline 3 & A laade thaph & Gavin + Lath & Gineve $=100 \%$ & 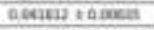 \\
\hline 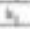 & Somin toinis & 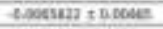 & 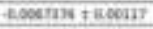 & 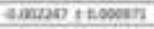 \\
\hline i & asules teove & 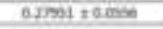 & anow acein & 6asese \pm 60000 \\
\hline
\end{tabular}

